\title{
Ridinilazole: a novel antimicrobial for Clostridium difficile infection
}

\author{
Jonathan C. Cho ${ }^{\text {a }}$, Matthew P. Crotty ${ }^{\text {, Joe Pardoc }}$
}

The University of Texas at Tyler, Tyler, TX; Methodist Dallas Medical Center, Dallas, TX; North FL/South GA Veterans Health System, Gainesville, FL, USA

\begin{abstract}
Clostridium difficile (C. difficile) infection remains a global healthcare threat worldwide and the limited options available for its treatment are of particular concern. Ridinilazole is one potential future agent, as it demonstrates rapid bactericidal activity against $C$. difficile. Current studies show that ridinilazole has a lower propensity for collateral damage to the gut microbiome and appears to diminish the production of $C$. difficile toxins. Results from phase II studies demonstrate that patients receiving ridinilazole had a higher sustained clinical response compared with patients receiving vancomycin $(66.7 \%$ vs. $42.4 \%$; $\mathrm{P}=0.0004)$. Adverse reactions were similar between ridinilazole and vancomycin ( $40 \%$ vs. $56 \%$, respectively), with most being gastrointestinal-related. Nausea $(20 \%)$ and abdominal pain (12\%) were the most commonly reported adverse reactions associated with ridinilazole. Phase II study results are promising and future availability of phase III trial results will help further delineate the role and value of ridinilazole.
\end{abstract}

Keywords Ridinilazole, Clostridium difficile, infectious diarrhea

Ann Gastroenterol 2019; 32 (X): 1-7

\section{Introduction}

Clostridium difficile (C. difficile) is recognized as an urgent threat to human health and represents an extremely challenging pathogen, given its impact on the healthcare system [1-5]. C. difficile infection (CDI) is recognized as the most common healthcare-associated infection and has spread across the globe [1,6-9]. Efforts in antimicrobial stewardship and infection prevention have demonstrated effectiveness in reducing the burden of CDI [10-13]. Unfortunately, CDI remains a looming presence in modern healthcare. One of the most challenging

${ }^{\mathrm{a} C}$ College of Pharmacy, The University of Texas at Tyler, Tyler, TX

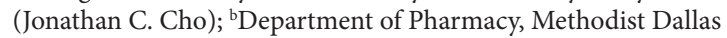
Medical Center, Dallas, TX (Matthew P. Crotty); ${ }^{\mathrm{c} D e p a r t m e n t ~ o f ~}$ Pharmacy, North FL/South GA Veterans Health System, Gainesville, FL (Joe Pardo), USA

Conflict of Interest: JCC serves on the speakers' bureau for Allergan. MPC served as a consultant for Theravance and Nabriva. The views expressed in this article are those of the authors and do not necessarily reflect the position or policy of the Department of Veterans Affairs or the United States government

Correspondence to: Jonathan C. Cho, Pharm D, MBA, BCPS, Clinical Assistant Professor, College of Pharmacy, The University of Texas at Tyler, 3900 University Blvd., Tyler TX, 75799, USA,

e-mail: jcho@uttyler.edu

Received 27 July 2018; accepted 7 November 2018; published online 13 December 2018

DOI: https://doi.org/10.20524/aog.2018.0336 aspects of managing patients with C. difficile is the pathogen's propensity to cause recurrent infections. Recurrence rates of up to $25 \%$ have been reported following treatment with metronidazole or vancomycin $[14,15]$. The vicious cycle of recurrence continues further, with patients who have one recurrence being at increased risk for another [16].

Treatment of acute CDI is currently limited to the antibiotics metronidazole, vancomycin and fidaxomicin, with bezlotoxumab being an option as adjunctive therapy. Metronidazole has largely fallen out of favor because of potential issues of resistance and inferior clinical response [17]. Vancomycin is currently utilized as first-line therapy; however, recurrence remains a substantial issue with this agent $[14,17,18]$. Fidaxomicin has demonstrated similar efficacy to that of vancomycin with the benefit of decreased recurrence; however, this agent may not be as effective against the fluoroquinolone-resistant BI/NAP1/027 strain (ribotype 027), the predominant ribotype in North America [19-21]. In view of the problematic nature of currently available treatment options, ridinilazole represents a potential welcome addition to the armamentarium. This paper will review the pharmacology, spectrum of activity, pharmacokinetics, pharmacodynamics, clinical trials and safety of ridinilazole.

\section{Data sources}

To gather relevant published information, a literature search was performed using PubMed, EMBSCO and Google Scholar electronic databases for relevant publications written 
in the English language. Search terms included ridinilazole, SMT19969, and C. difficile. Information was also gathered from abstracts from the Interscience Conference on Antimicrobial Agents and Chemotherapy, IDWeek, American Society for Microbiology Microbe, and the European Congress of Clinical Microbiology and Infectious Diseases.

\section{Chemistry and pharmacology}

Ridinilazole, previously known as SMT19969, is a novel antibacterial currently under development for the treatment of $C$. difficile. Its chemical name is $2,2^{\prime}$-bis(4-pyridyl) $3 \mathrm{H}, 3^{\prime} \mathrm{H}$ $5,5^{\prime}$-bibenzimidazole. The agent has a unique mechanism of action andisthought tointerferewith celldivision. It demonstrates rapid bactericidal activity against $C$. difficile $[22,23]$. A cluster of $C$. difficile genes involved in cell division were found to display an altered expression following exposure to ridinilazole in a transcriptomic analysis. These findings, combined with previous work demonstrating a filamentous phenotype of C. difficile upon exposure to ridinilazole, suggest that the agent works by targeting cell growth [22,24]. Preclinical animal studies demonstrated negligible systemic exposure of the orally administered agent [25]. Following oral administration of $200 \mathrm{mg}$ b.i.d., ridinilazole is minimally absorbed from the gastrointestinal lumen, resulting in low systemic levels and high fecal concentrations [26]. The specificity of activity targeting C. difficile and limiting collateral damage to the gut microbiota, along with its minimal systemic absorption, make ridinilazole an exciting agent for treating CDI.

\section{Microbiologic activity}

\section{Activity against $C$. difficile}

Ridinilazole has demonstrated potent activity against a breadth of $C$. difficile strains in vitro [27-32]. In a study of 107 C. difficile strains of varying resistance phenotypes, ridinilazole demonstrated minimum inhibitory concentration (MIC) values (MIC range: $0.015-0.5 \mathrm{mg} / \mathrm{L} ; \mathrm{MIC}_{50}: 0.03 \mathrm{mg} / \mathrm{L}$; $\mathrm{MIC}_{90}: 0.125 \mathrm{mg} / \mathrm{L}$ ) lower than those of metronidazole (MIC range: $\left.<0.125-2 \mathrm{mg} / \mathrm{L} ; \mathrm{MIC}_{50}: 0.5 \mathrm{mg} / \mathrm{L} ; \mathrm{MIC}_{90}: 2 \mathrm{mg} / \mathrm{L}\right)$ and vancomycin (MIC range: $0.5-8 \mathrm{mg} / \mathrm{L} ; \mathrm{MIC}_{50}: 1 \mathrm{mg} / \mathrm{L}$; $\mathrm{MIC}_{90}: 2 \mathrm{mg} / \mathrm{L}$ ), and comparable to those of fidaxomicin (MIC range: 0.004-0.125 $\mathrm{mg} / \mathrm{L} ; \quad \mathrm{MIC}_{50}: 0.06 \mathrm{mg} / \mathrm{L}$; $\left.\mathrm{MIC}_{90}: 0.125 \mathrm{mg} / \mathrm{L}\right)$ [29]. Among 200 isolates collected from C. difficile toxin-positive stool samples at 6 United States sites, ridinilazole was similarly found to have lower MIC values (MIC range: $0.12-0.5 \mathrm{mg} / \mathrm{L} ; \mathrm{MIC}_{50}: 0.12 \mathrm{mg} / \mathrm{L} ; \mathrm{MIC}_{90}: 0.25 \mathrm{mg} / \mathrm{L}$ ) than metronidazole (MIC range: $0.12-2 \mathrm{mg} / \mathrm{L} ; \mathrm{MIC}_{50}: 0.25 \mathrm{mg} / \mathrm{L}$; $\mathrm{MIC}_{90}: 1 \mathrm{mg} / \mathrm{L}$ ) or vancomycin (MIC range: $0.25-4 \mathrm{mg} / \mathrm{L}$; $\left.\mathrm{MIC}_{50}: 1 \mathrm{mg} / \mathrm{L} ; \mathrm{MIC}_{90}: 2 \mathrm{mg} / \mathrm{L}\right)$ and was comparable to fidaxomicin (MIC range: $0.015-1 \mathrm{mg} / \mathrm{L} ; \mathrm{MIC}_{50}: 0.03 \mathrm{mg} / \mathrm{L}$; $\left.\mathrm{MIC}_{90}: 0.125 \mathrm{mg} / \mathrm{L}\right)[30]$.

Mutant prevention concentrations, used to describe the antimicrobial drug concentration required to block the growth of the least susceptible cell present in a high-density bacterial population and give insight into the likelihood for resistance selection compared with achievable therapeutic concentrations, remained well below $(\leq 0.25 \mathrm{mg} / \mathrm{L})$ fecal concentrations of ridinilazole, suggesting a low propensity for resistance selection among $C$. difficile strains clinically [33]. Following 14 serial passages at sub-MIC concentrations $(0.5$ $\times$ MIC), no increase in ridinilazole MIC was noted and spontaneous mutations were highly infrequent, occurring at $<3.17 \times 10^{-9}$ [34]. Moreover, no spontaneously ridinilazoleresistant mutants were identified among 2 clinical strains of C. difficile (ribotype 012 and ribotype 027) following 15 serial passages using brain heart infusion broth containing ridinilazole concentrations ranging from 0.004-64 mg/L [35]. In contrast, Bassères et al were able to isolate a stable mutant of C. difficile ribotype 027 with a filamentous phenotype following 11 serial passages that stabilized at an MIC of $0.48 \mathrm{mg} / \mathrm{L}$, eight times higher than the initial value of the isolate $(0.06 \mathrm{mg} / \mathrm{L})$ [36]. Overall, the development of $C$. difficile resistance to ridinilazole seems likely to be uncommon.

\section{Activity against gut microbiota}

While exhibiting substantial activity against C. difficile, ridinilazole is largely specific for this bacterium and demonstrates limited activity against gut microbiota. When tested in vitro against 350 bacterial isolates representing common intestinal flora, ridinilazole showed limited activity against gramnegative and gram-positive anaerobes other than Clostridium spp., summarized in Table 1. Of note is the demonstrated lack of activity of ridinilazole against Bacteroides fragilis (B. fragilis) (MIC range: 512 to $>512 \mathrm{mg} / \mathrm{L} ; \mathrm{MIC}_{50}:>512 \mathrm{mg} / \mathrm{L}$ ) and Bifidobacteria spp. (MIC range: 16 to $>512 \mathrm{mg} / \mathrm{L} ; \mathrm{MIC}_{50}$ : $>512 \mathrm{mg} / \mathrm{L}$ ). In contrast, vancomycin MICs for B. fragilis and Bifidobacteria spp. ranged from $32-128 \mathrm{mg} / \mathrm{L}$ and $0.5-1 \mathrm{mg} / \mathrm{L}$, respectively [27]. In a subsequent evaluation of 162 strains representing 35 species of Clostridium spp. (non-C. difficile), ridinilazole showed variable activity MIC range (0.06 to $>512 \mathrm{mg} / \mathrm{L}$ ) depending upon species and strain [37]. In an in vitro CDI human gut model, ridinilazole demonstrated minimal impact on viable cell counts of indigenous gut microbiota other than total Clostridium spp. [32].

In fecal samples from healthy adult males who were given ridinilazole 200 or $500 \mathrm{mg}$ b.i.d. in a Phase I trial, clostridial viable counts were below the lower limits of detection for viable counting and other gut microbiota were largely unchanged [38]. Further phase I evaluation similarly showed a limited impact of ridinilazole on gut microbiota other than Clostridium spp. Counts of Bacteroides spp. increased ( $2 \log$ by day 9), while counts of lactobacilli decreased ( $1 \log$ by day 4 , remaining constant until day 9). Minimal change was observed in counts of Bifidobacterium spp. and total anaerobes, while counts of total aerobes and lactose fermenting Enterobacteriaceae increased (2 log by day 9) [26]. An evaluation of fecal samples from 82 patients enrolled in a phase II trial similarly demonstrated a limited impact of ridinilazole on gut microbiota, except for a 1 $\log _{10}$ reduction on day 10 of therapy for Clostridium leptum. In 
Table 1 In vitro susceptibility of various gastrointestinal bacteria to ridinilazole and select comparator agents [27,34]

\begin{tabular}{|c|c|c|c|c|}
\hline Ribotype & Agent & MIC range $(\mathrm{mg} / \mathrm{L})$ & $\mathrm{MIC}_{50}(\mathrm{mg} / \mathrm{L})$ & $\mathrm{MIC}_{90}(\mathrm{mg} / \mathrm{L})$ \\
\hline \multirow[t]{4}{*}{ Bifidobacterium species } & Ridinilazole & $16 \rightarrow 512$ & $64,>512$ & $128,>512$ \\
\hline & Fidaxomicin & $\geq 0.03-0.25$ & 0.125 & 0.125 \\
\hline & Vancomycin & $0.5-1$ & 1 & 1 \\
\hline & Metronidazole & $2 \rightarrow 512$ & 32 & 128 \\
\hline \multirow[t]{4}{*}{ Bacteroides fragilis } & Ridinilazole & $512 \rightarrow 512$ & $>512$ & $>512$ \\
\hline & Fidaxomicin & $>512$ & $>512$ & $>512$ \\
\hline & Vancomycin & $32-128$ & 64 & 64 \\
\hline & Metronidazole & $0.5-2$ & 1 & 2 \\
\hline \multirow[t]{4}{*}{ Clostridium inпосиuт } & Ridinilazole & $0.06-1$ & 0.25 & 1 \\
\hline & Fidaxomicin & $128-512$ & 256 & 256 \\
\hline & Vancomycin & 16 & 16 & 16 \\
\hline & Metronidazole & $0.5-16$ & 1 & 2 \\
\hline \multirow[t]{4}{*}{ Clostridium ramosum } & Ridinilazole & $128 \rightarrow 512$ & $>512$ & $>512$ \\
\hline & Fidaxomicin & $>512$ & $>512$ & $>512$ \\
\hline & Vancomycin & 4 & 4 & 4 \\
\hline & Metronidazole & $0.5-8$ & 0.5 & 1 \\
\hline \multirow[t]{4}{*}{ Enterococcus faecalis } & Ridinilazole & $128 \rightarrow 512$ & $>512$ & $>512$ \\
\hline & Fidaxomicin & $1-8$ & 8 & 8 \\
\hline & Vancomycin & $1-4$ & 1 & 4 \\
\hline & Metronidazole & $>512$ & $>512$ & $>512$ \\
\hline \multirow[t]{4}{*}{ Enterococcus faecium } & Ridinilazole & $64 \rightarrow 512$ & 128 & 128 \\
\hline & Fidaxomicin & $0.5-16$ & 8 & $>128$ \\
\hline & Vancomycin & $0.5-256$ & 0.5 & 256 \\
\hline & Metronidazole & $256 \rightarrow 512$ & $>512$ & $>512$ \\
\hline \multirow[t]{4}{*}{ Lactobacillus species } & Ridinilazole & $0.06 \rightarrow 512$ & 16,128 & $>512$ \\
\hline & Fidaxomicin & $0.25 \rightarrow 512$ & 8 & $>512$ \\
\hline & Vancomycin & $0.5 \rightarrow 512$ & 256 & $>512$ \\
\hline & Metronidazole & $2 \rightarrow 512$ & $>512$ & $>512$ \\
\hline \multirow[t]{4}{*}{ Peptostreptococcus anaerobius } & Ridinilazole & $0.125-128$ & 64 & 64 \\
\hline & Fidaxomicin & $\geq 0.03$ & $\geq 0.03$ & $\geq 0.03$ \\
\hline & Vancomycin & 0.5 & 0.5 & 0.5 \\
\hline & Metronidazole & $0.125-1$ & 0.5 & 1 \\
\hline \multirow[t]{4}{*}{ Prevotella species } & Ridinilazole & $32 \rightarrow 512$ & $>512$ & $>512$ \\
\hline & Fidaxomicin & $64 \rightarrow 512$ & $>512$ & $>512$ \\
\hline & Vancomycin & $64 \rightarrow 512$ & 128 & 512 \\
\hline & Metronidazole & $0.25-1$ & 0.5 & 1 \\
\hline
\end{tabular}

contrast, vancomycin exposure led to significantly decreased bacterial counts of Bacteroides spp., Prevotella spp., Clostridium coccoides and Clostridium leptum from day 1 to 10 by approximately $3 \log _{10}$ copies per gram of stool. This substantial impact led to increased bacterial counts of Enterobacteriaceae during vancomycin treatment, but not during ridinilazole treatment [39]. A separate exploratory phase II trial evaluated the impact of ridinilazole $(n=14)$ and fidaxomicin $(n=13)$ on the host gut microbiome in a randomized, open label study. The results suggested that ridinilazole was more preserving of the gut microbiome compared with fidaxomicin. Specifically, fidaxomicin exposure led to a reduction in 10 of the bacterial families analyzed compared to only 2 bacterial families with reduced populations among samples exposed to ridinilazole. 
Moreover, fidaxomicin exposure led to a reduction in the population of bacterial families belonging to the Firmicutes, thought to have a role in protection against CDI [40]. An additional phase II trial compared the composition of fecal microbiota between patients administered ridinilazole and vancomycin using $16 \mathrm{~S} \mathrm{rDNA}$ at baseline, day 5 of therapy, day 10 (end of therapy), day 25 and at the end of the study. Significant reductions in percent relative abundance were modest and were only found within the Firmicutes phylum among samples from ridinilazole-exposed patients. Within the Firmicutes, vancomycin affected more taxa than ridinilazole. Additionally, vancomycin exposure led to substantial decreases in the abundance of Actinobacteria, Bacteroidetes, and Proteobacteria. Interestingly the fecal microbiota returned to baseline more quickly among ridinilazole-treated patients [41]. The targeted activity of ridinilazole has demonstrated a lower propensity for collateral damage to the gut microbiome in multiple models and human fecal evaluations and highlights a promising aspect of this new agent.

\section{Pharmacokinetics}

A phase I study $(n=56)$ was conducted to elucidate the pharmacokinetics and safety of ridinilazole in healthy volunteers after single and multiple oral doses taken with or without food [26]. All doses studied were well-tolerated and any adverse effects potentially due to ridinilazole were mild. In part one, placebo or single ascending doses of ridinilazole $(2,20,100,400,1000$ or $2000 \mathrm{mg})$ were administered. Plasma levels following doses up to $2000 \mathrm{mg}$ in the fasted state were generally undetectable. The $1000 \mathrm{mg}$ dose was studied in the fasted and fed state. A single oral dose of $1000 \mathrm{mg}$ in the fed state produced low but detectable plasma concentrations in all 6 subjects in that study arm (range: 0.102-0.296 ng/mL). Maximum plasma concentrations were observed $4 \mathrm{~h}$ following administration. In part two, ridinilazole was administered in the fed state at a dose of $200 \mathrm{mg}$ or $500 \mathrm{mg}$ b.i.d. for 10 days. By Day 10, low plasma ridinilazole concentrations were observed in most test subjects (range: $0.105-0.305 \mathrm{ng} / \mathrm{mL}$ ) with $\mathrm{T}_{\max }$ again occurring at 4 hours post-dose. In those receiving $200 \mathrm{mg}$ b.i.d., the mean area under the plasma concentration-time curve from time zero up to the last quantifiable concentration was $0.670 \mathrm{ng} . \mathrm{h} / \mathrm{mL}$ (range: $0.524-1.30$ ), with average maximum plasma concentrations of $0.141 \mathrm{ng} / \mathrm{mL}$ (range: $0.108-0.243$ ). These findings are consistent with those of prior animal studies and subsequent phase II human data, collectively indicating that ridinilazole is mostly contained within the gastrointestinal tract following oral administration [42-44].

Fecal concentrations of ridinilazole were also measured in the phase I study. Ridinilazole was primarily excreted in the feces as unchanged parent drug, which accounted for $97 \%$ of the total peak area. Mean fecal concentrations increased with increasing doses. For subjects receiving $200 \mathrm{mg}$ b.i.d., mean (range) fecal concentrations on Day 5 and Day 10 were $1466 \mu \mathrm{g} / \mathrm{g}(847-2390 \mu \mathrm{g} / \mathrm{g})$ and $1364 \mu \mathrm{g} / \mathrm{g}(783-1980 \mu \mathrm{g} / \mathrm{g})$, respectively [26]. Similar fecal concentrations were observed in a phase II trial, wherein the mean fecal concentration on treatment day 10 was $1373 \mu \mathrm{g} / \mathrm{g}$ [44]. The observed fecal concentrations across clinical and preclinical studies are significantly above the $\mathrm{MIC}_{90}$ of ridinilazole for C. difficile (0.125-0.25 mg/L) [29,30].

\section{Pharmacodynamics}

C. difficile isolates exposed to ridinilazole exhibit elongated morphology. Though exposed cells may continue to replicate DNA initially, septum formation is halted, which ultimately impairs cell division [22]. The pharmacodynamic effects of ridinilazole have been studied in vitro against a multitude of C. difficile strains. Ridinilazole was tested against 82 clinical C. difficile isolates collected in the United Kingdom [28]. The $\mathrm{MIC}_{90}$ was $0.125 \mathrm{mg} / \mathrm{L}, 16$ - to 32 -fold lower than $\mathrm{MIC}_{90}$ values for metronidazole and vancomycin. Killing kinetics were compared against fidaxomicin and vancomycin for three different $C$. difficile strains: BI1 (ribotype 027), 630 (ribotype 012) and 5325 (ribotype 078). For the BI1 strain, vancomycin was bactericidal at $2 \times$ MIC. Fidaxomicin was bacteriostatic at $1-10 \times$ MIC $\left(1.5-2.0 \log _{10}\right.$ reduction at $\left.24 \mathrm{~h}\right)$ and bactericidal at $20 \times$ MIC. Ridinilazole was bactericidal at all concentrations tested, with reduction in viable $C$. difficile counts to below the limit of detection by $24 \mathrm{~h}$. Killing by ridinilazole did not appear dependent on drug concentration. Ridinilazole was bactericidal against strains 630 and 5325 at $\geq 5 \times$ MIC and $\geq 10 \times$ MIC, respectively. The post-antibiotic effect (PAE) for ridinilazole against all strains was $>20 \mathrm{~h}$ at $20 \times$ MIC, a concentration that should be exceeded in patients taking $200 \mathrm{mg}$ by mouth b.i.d. [26,44]. Fidaxomicin also exhibited a PAE for all strains, while vancomycin was generally bacteriostatic with minimal PAE (0-2 h) [28].

Bassères et al examined in vitro production of toxins $\mathrm{A}$ and $\mathrm{B}$ in $\mathrm{C}$. difficile strains exposed to sub-MIC $(0.125 \times-0.5 \times)$ and supra-MIC $(4 \times$ or $40 \times$ MIC) concentrations of ridinilazole [22]. Toxin $\mathrm{B}$ production was suppressed below the limit of detection with all concentrations of ridinilazole tested. Toxin A levels were reduced by $75 \%$ to $>90 \%$ at varying ridinilazole concentrations. Cytotoxin levels were similarly reduced in an in vitro gut model of CDI [32]. Additionally, interleukin (IL)-8 release from eukaryotic cell lines exposed to antibiotic-treated $C$. difficile isolates were measured [22]. IL-8 production was reduced by $25-74 \%$ following exposure to $C$. difficile that had been treated with ridinilazole, suggesting that ridinilazole-mediated toxin reduction may dampen host inflammatory responses. Metronidazole and vancomycin had minimal impact on toxin production, and IL-8 production after exposure to metronidazole or vancomycin was similar to that of controls [22]. In a phase II study, 100 patients with CDI were randomized to 10 days of treatment with either ridinilazole or vancomycin and fecal concentrations of calprotectin and lactoferrin were measured to determine bowel inflammation at baseline, day 5, and day 10 (end of treatment) [45]. When all disease severities were analyzed together at day 10 , concentrations of both toxins were reduced similarly from baseline in both the ridinilazole and 
vancomycin groups. In contrast, ridinilazole demonstrated a greater reduction in lactoferrin (1.93 vs. $\left.0.62 \log _{10}\right)$ and calprotectin (1.70 vs. $0.22 \log _{10}$ ) from baseline to day 10 when only severe disease was analyzed [45].

The efficacy of ridinilazole versus comparator agents has also been investigated in animal models. The hamster model of clindamycin-induced CDI is a validated method for evaluating the potential of new CDI therapies [46]. In this model, hamsters are rendered susceptible to CDI by oral administration of clindamycin followed by infection with $C$. difficile spores. This protocol produces a severe model of CDI with subject death generally occurring within 3 days in the absence of treatment. In two hamster-model experiments using the epidemic BI/ NAP1 (ribotype 025) strain, survival rates with ridinilazole treatment at a total daily dose of $50 \mathrm{mg} / \mathrm{kg}$ were $70 \%$ and $100 \%$ vs. $60 \%$ and $10 \%$ with vancomycin $[42,43]$. Fidaxomicin was also effective against the epidemic strain, with $80-90 \%$ of animals surviving [42]. In a study arm using the non-epidemic VA11 clinical strain [43], ridinilazole was associated with better survival rates than vancomycin ( $80-95 \%$ vs. $50 \%)$. In a study using the non-epidemic ribotype 012 strain [42], all vancomycin-treated animals survived during dosing, but there was a $0 \%$ survival rate in the follow-up period. Fidaxomicin conferred some protection, but overall survival rates across different fidaxomicin dosing groups were low, ranging from $0-40 \%$. Ridinilazole conferred significant protection against ribotype 012 , with survival rates of $80-100 \%$ across dosing groups. Further, following administration of high ridinilazole doses $(25 \mathrm{mg} / \mathrm{kg}$ b.i.d.), no spores were isolated in animals with ribotype 027 infection from day 7 onwards, although a significant number of animals had spores isolated in the ribotype 012 arm [42].

\section{Safety and efficacy}

The safety and efficacy of ridinilazole were compared to those of vancomycin in a phase II, double-blind, activecontrolled study at 33 various sites in the United States and Canada [44]. Patients were included in the study if they met the definition of having CDI, had a positive diagnostic test for C. difficile or had received more than $24 \mathrm{~h}$ of CDI treatment prior to initiation of the study agent. Included patients were randomized 1:1 to receive either ridinilazole $200 \mathrm{mg}$ orally b.i.d. or vancomycin $125 \mathrm{mg}$ orally q.i.d. for 10 days. The primary endpoint was sustained clinical response, which was defined as clinical cure at test of cure (TOC) (day 12-14) and absence of CDI recurrence 30 days after end of treatment (day 10-11). Secondary endpoints and objectives included time to hospital discharge, time to diarrhea resolution, and tolerability of ridinilazole compared with vancomycin. The primary efficacy analysis was performed on 69 patients in the modified intent-to-treat (mITT) population. Patients receiving ridinilazole had a higher sustained clinical response compared with patients receiving vancomycin in the mITT population ( $66.7 \%$ vs. $42.4 \% ; \mathrm{P}=0.0004)$. Ridinilazole was determined to be non-inferior to vancomycin; 36 (77.8\%) and
$23(67.7 \%)$ patients in the ridinilazole and vancomycin groups, respectively, had a clinical response at TOC. The median time to diarrhea resolution was 4 and 5 days (hazard ratio [HR] $1.19 ; 90 \%$ confidence interval [CI] 0.76-1.87) and median time to hospital discharge was 5 and 7 days (HR 0.99; 90\%CI 0.342.91) for the ridinilazole and vancomycin groups, respectively. CDI recurrence was documented in $14.3 \%$ of patients receiving ridinilazole and $34.8 \%$ of patients receiving vancomycin $(-16.2 \%, 90 \%$ CI -35.5 to 3$)$.

Ridinilazole displayed adverse reactions similar to those of vancomycin, with most being gastrointestinal-related (40\% vs. $56 \%$, respectively) [44]. Three patients had severe adverse reactions that may have been related to the study drug: one patient in the ridinilazole arm had hypokalemia and two patients in the vancomycin arm had serious reactions: one septic shock and moderate hematemesis and the other elevated liver enzymes and diarrhea. Nausea (20\%) and abdominal pain (12\%) were the most commonly reported adverse reactions associated with ridinilazole. These results were similar to those seen in phase I studies [26].

\section{Concluding remarks}

C. difficile infection remains a global healthcare threat with a propensity to cause recurrent disease. Currently, treatment options for C. difficile are limited and ridinilazole seems to be a promising potential agent for the treatment of CDI. Ridinilazole has also demonstrated a lower propensity for collateral damage to the gut microbiome and appears to diminish the production of $C$. difficile toxins and subsequent bowel inflammation, which may prove advantageous in managing severe CDI. Current phase II results are promising and future availability of phase III trial results will help further delineate the role and value of ridinilazole.

\section{References}

1. Kyne L, Hamel MB, Polavaram R, Kelly CP. Health care costs and mortality associated with nosocomial diarrhea due to Clostridium difficile. Clin Infect Dis 2002;34:346-353.

2. O'Brien JA, Lahue BJ, Caro JJ, Davidson DM. The emerging infectious challenge of Clostridium difficile-associated disease in Massachusetts hospitals: clinical and economic consequences. Infect Control Hosp Epidemiol 2007;28:1219-1227.

3. Dubberke ER, Olsen MA. Burden of Clostridium difficile on the healthcare system. Clin Infect Dis 2012;55 Suppl 2:S88-S92.

4. Centers for Disease Control and Prevention. Antibiotic resistance threats in the United States 2013. http://www.cdc.gov/drugresistance/ pdf/ar-threats-2013-508.pdf [Accessed November 11, 2018].

5. Lessa FC, Mu Y, Bamberg WM, et al. Burden of Clostridium difficile infection in the United States. N Engl J Med 2015;372:825-834.

6. He M, Miyajima F, Roberts P, et al. Emergence and global spread of epidemichealthcare-associated Clostridium difficile. Nat Genet 2013; 45:109-113.

7. Jones AM, Kuijper EJ, Wilcox MH. Clostridium difficile: a European perspective. J Infect 2013;66:115-128. 
8. Burke KE, Lamont JT. Clostridium difficile infection: a worldwide disease. Gut Liver 2014;8:1-6.

9. Borren NZ, Ghadermarzi S, Hutfless S, Ananthakrishnan AN. The emergence of Clostridium difficile infection in Asia: A systematic review and meta-analysis of incidence and impact. PLoS One 2017; 12:e176797.

10. Climo MW, Israel DS, Wong ES, Williams D, Coudron P, Markowitz SM. Hospital-wide restriction of clindamycin: effect on the incidence of Clostridium difficile-associated diarrhea and cost. Ann Intern Med 1998;128:989-995.

11. Valiquette L, Cossette B, Garant MP, Diab H, Pépin J. Impact of a reduction in the use of high-risk antibiotics on the course of an epidemic of Clostridium difficile-associated disease caused by the hypervirulent NAP1/027 strain. Clin Infect Dis 2007;45 Suppl 2:S112-S121.

12. Dingle KE, Didelot X, Quan TP, et al; Modernising Medical Microbiology Informatics Group. Effects of control interventions on Clostridium difficile infection in England: an observational study. Lancet Infect Dis 2017;17:411-421.

13. Baur D, Gladstone BP, Burkert F, et al. Effect of antibiotic stewardship on the incidence of infection and colonisation with antibioticresistant bacteria and Clostridium difficile infection: a systematic review and meta-analysis. Lancet Infect Dis 2017;17:990-1001.

14. Eyre DW, Walker AS, Wyllie D, et al; Infections in Oxfordshire Research Database. Predictors of first recurrence of Clostridium difficile infection: implications for initial management. Clin Infect Dis 2012;55(Suppl 2):S77-S87.

15. Eyre DW, Cule ML, Wilson DJ, et al. Diverse sources of C. difficile infectionidentified on whole-genomesequencing. NEnglJMed 2013; 369:1195-1205.

16. Kelly CP. Can we identify patients at high risk of recurrent Clostridium difficile infection? Clin Microbiol Infect 2012; 18(Suppl 6):21-27.

17. McDonald LC, Gerding DN, Johnson S, et al. Clinical Practice Guidelines for Clostridium difficile Infection in Adults and Children: 2017 Update by the Infectious Diseases Society of America (IDSA) and Society for Healthcare Epidemiology of America (SHEA). Clin Infect Dis 2018;66:987-994.

18. Debast SB, Bauer MP, Kuijper EJ. European Society of Clinical Microbiology and Infectious Diseases: update of the treatment guidance document for Clostridium difficile infection. Clin Microbiol Infect 2014;20(Suppl 2):1-26.

19. Louie TJ, Miller MA, Mullane KM, et al; OPT-80-003 Clinical Study Group. Fidaxomicin versus vancomycin for Clostridium difficile infection. N Engl J Med 2011;364:422-431.

20. Crook DW, Walker AS, Kean Y, et al; Study 003/004 Teams. Fidaxomicin versus vancomycin for Clostridium difficile infection: meta-analysis of pivotal randomized controlled trials. Clin Infect Dis 2012;55(Suppl 2):S93-S103.

21. Tickler IA, Goering RV, Whitmore JD, Lynn AN, Persing DH, Tenover FC; Healthcare Associated Infection Consortium. Strain types and antimicrobial resistance patterns of Clostridium difficile isolates from the United States, 2011 to 2013. Antimicrob Agents Chemother 2014;58:4214-4218.

22. Bassères E, Endres BT, Khaleduzzaman M, et al. Impact on toxin production and cell morphology in Clostridium difficile by ridinilazole (SMT19969), a novel treatment for C. difficile infection. $J$ Antimicrob Chemother 2016;71:1245-1251.

23. Vickers RJ, Tillotson G, Goldstein EJ, Citron DM, Garey KW, Wilcox MH. Ridinilazole: a novel therapy for Clostridium difficile infection. Int J Antimicrob Agents 2016;48:137-143.

24. Bassères E, Endres BT, Vickers R, et al. Transcriptome functional analysis of Clostridium difficile exposed to ridinilazole; insight into potential mechanism of action. In: Abstracts of the Twenty-seventh European Congress of Clinical Microbiology and Infectious
Diseases, Vienna, Austria, 2017. Abstract EP0404, Blackwell, Hoboken, NJ, USA.

25. Vickers RJ, Storer R, Tinsley J, et al. SMT19969: Preclinical safety and pharmacokinetics of a novel antibiotic for Clostridium difficile infection. In: Abstracts of the Twenty-third European Congress of Clinical Microbiology and Infectious Diseases, Berlin, Germany, 2013, Abstract P1656.

26. Vickers R, Robinson N, Best E, Echols R, Tillotson G, Wilcox M. A randomised phase 1 study to investigate safety, pharmacokinetics and impact on gut microbiota following single and multiple oral doses in healthy male subjects of SMT19969, a novel agent for Clostridium difficile infections. BMC Infect Dis 2015;15:91.

27. Goldstein EJ, Citron DM, Tyrrell KL, Merriam CV. Comparative in vitro activities of SMT19969, a new antimicrobial agent, against Clostridium difficile and 350 gram-positive and gram-negative aerobic and anaerobic intestinal flora isolates. Antimicrob Agents Chemother 2013;57:4872-4876.

28. Corbett D, Wise A, Birchall S, et al. In vitro susceptibility of Clostridium difficile to SMT19969 and comparators, as well as the killing kinetics and post-antibiotic effects of SMT19969 and comparators against C. difficile. J Antimicrob Chemother 2015; 70:1751-1756.

29. Freeman J, Vernon J, Vickers R, Wilcox MH. Susceptibility of Clostridium difficile isolates of varying antimicrobial resistance phenotypes to smt19969 and 11 comparators. Antimicrob Agents Chemother 2016;60:689-692.

30. Snydman DR, McDermott LA, Thorpe CM, et al. In-vitro activity of ridinilazole and comparators against isolates of Clostridium difficile obtained from stools of patients as part of US Surveillance in 2014. In: American Society for Microbiology Microbe 2017, New Orleans, LA, USA, Abstract 235.

31. Snydman DR, McDermott LA, Thorpe CM, et al. Antimicrobial susceptibility and ribotypes of Clostridium difficile isolates from a Phase 2 clinical trial of ridinilazole (SMT19969) and vancomycin. J Antimicrob Chemother 2018;73:2078-2084.

32. Baines SD, Crowther GS, Freeman J, Todhunter S, Vickers R, Wilcox MH. SMT19969 as a treatment for Clostridium difficile infection: an assessment of antimicrobial activity using conventional susceptibility testing and an in vitro gut model. J Antimicrob Chemother 2015;70:182-189.

33. Blondeau JM, Shebelski SD, Vickers R. Mutant prevention concentration of SMT19969 against clinical isolates of Clostridium difficile. In: Abstracts of the Fifty-fourth Interscience Conference on Antimicrobial Agents and Chemotherapy, Washington DC, USA, 2014, Abstract F-245.

34. Vickers R, Tinsley J, Wilson F, et al. SMT19969: In vitro and in vivo evaluation of a novel antibiotic for Clostridium difficile infection. In: Abstracts of the Twenty-third European Congress of Clinical Microbiology and Infectious Diseases, Berlin, Germany, 2013, Abstract P1655.

35. Hall D, Hinshaw R, Pillar C, et al. Resistance development studies with ridinilazole (RDZ) and Clostridium difficile. In: American Society for Microbiology Microbe 2016, Boston, MA, USA, Abstract 443.

36. Bassères E, Rashid T, Endres BT, et al. In vitro selection of Clostridium difficile resistance mutants exposed to ridinilazole. In: American Society for Microbiology Microbe 2017, New Orleans, LA, USA, Abstract 233.

37. Goldstein EJ, Citron DM, Tyrrell KL. Comparative in vitro activities of SMT19969, a new antimicrobial agent, against 162 strains from 35 less frequently recovered intestinal Clostridium species: implications for Clostridium difficile recurrence. Antimicrob Agents Chemother 2014;58:1187-1191.

38. Best E, Vickers R, Wilcox MH. Effects of a novel antimicrobial (SMT19969) for treatment of Clostridium difficile infection (CDI) 
on gut flora. In: Abstracts of the Fifty-third Interscience Conference on Antimicrobial Agents and Chemotherapy, Denver, Colorado, 2013, Abstract K-167.

39. Chang J, Kane A, Snydman DR, et al. Ridinilazole preserves major components of the intestinal microbiota during treatment of Clostridium difficile infection. In: American Society for Microbiology Microbe 2016, Boston, MA, USA, Abstract LB-116.

40. Mitra S, Chilton C, Freeman J, et al. Preservation of gut microbiome following ridinilazole versus fidaxomicin treatment of Clostridium difficile infection. In: ID Week, San Diego, CA, 2017, Abstract 1842.

41. Thorpe C, Kane A, Chang J, et al. Ridinilazole treatment for C. difficile infection preserves the intestinal microbiota of patients. In: Abstracts of the Twenty-seventh European Congress of Clinical Microbiology and Infectious Diseases, Vienna, Austria, 2017, Abstract P2049.

42. Sattar A, Thommes P, Payne L, Warn P, Vickers RJ. SMT19969 for Clostridium difficile infection (CDI): in vivo efficacy compared with fidaxomicin and vancomycin in the hamster model of CDI. J Antimicrob Chemother 2015;70:1757-1762.

43. Weiss W, Pulse M, Vickers R. In vivo assessment of SMT19969 in a hamster model of Clostridium difficile infection. Antimicrob Agents Chemother 2014;58:5714-5718.

44. Vickers RJ, Tillotson GS, Nathan R, et al; CoDIFy study group. Efficacy and safety of ridinilazole compared with vancomycin for the treatment of Clostridium difficile infection: a phase 2, randomised, double-blind, active-controlled, non-inferiority study. Lancet Infect Dis 2017;17:735-744.

45. Vicker RJ, Yan K, Li Y. Ridinilazole for Clostridium difficile infection (CDI) - reductions in calprotectin and lactoferrin during therapy. In: American Society for Microbiology Microbe 2016, Boston, MA, USA, Abstract 441.

46. Douce G, Goulding D. Refinement of the hamster model of Clostridium difficile disease. Methods Mol Biol 2010;646:215-227. 